\title{
Metastatic Lymph Node Ratio of Central Neck Compartment Has Predictive Values for Locoregional Recurrence in Papillary Thyroid Microcarcinoma
}

\author{
Sung Yong Choi ${ }^{1, *} \cdot$ Jae-Keun $\mathrm{Cho}^{2, \star} \cdot$ Jeong Hwan Moon ${ }^{3} \cdot$ Young-lk Son ${ }^{1}$ \\ ${ }^{1}$ Department of Otorhinolaryngology-Head and Neck Surgery, Samsung Medical Center, Sungkyunkwan University School of Medicine, Seoul; \\ ${ }^{2}$ Department of Otorhinolaryngology-Head and Neck Surgery, Inje University Ilsan Paik Hospital, Inje University College of Medicine, Goyang; \\ ${ }^{3}$ Department of Otorhinolaryngology-Head and Neck Surgery, Dankook University College of Medicine, Cheonan, Korea
}

Objectives. This study aimed to evaluate the significance of metastatic lymph node ratio (the ratio between the metastatic lymph node and the harvested lymph nodes; MLNR) in the central neck for the prediction of locoregional recurrence in patients with papillary thyroid microcarcinoma.

Methods. After reviewing medical records of papillary thyroid microcarcinoma patients who received total thyroidectomy with central neck node dissection, 573 consecutive adult patients were enrolled in this study, with a follow-up period of more than 36 months. Regarding the risk of recurrence, multivariate analyses were performed with the following variables; sex, age, multiplicity of the primary tumor, presence of pathological extrathyroidal extension, the level of postoperative stimulated serum thyroglobulin, the number of harvested lymph nodes, the number of lymph node metastasis and MLNR.

Results. The MLNR showed a predictive significance for the locoregional recurrence $(P<0.05)$. Most recurrences were occurred in the lateral neck $(\mathrm{n}=12,80 \%)$ with a median interval of 20 months. The lowest cutoff value of the MLNR for a meaningful separation of disease recurrence was 0.44 (hazard ratio, 8.86; $95 \%$ confidence interval, 1.49 to 52.58; $P=0.001)$.

Conclusion. When the MLNR is higher than 0.44 , there is an increased risk of locoregional recurrence mostly in the lateral neck. Therefore, MLNR of the central neck in a permanent or frozen biopsy may be helpful in decision making in the extent of thyroidectomy and/or the need for contralateral central neck lymph nodes dissection.

Keywords. Papillary Thyroid Microcarcinoma; Lymphatic Metastasis; Neck Dissection; Neoplasm Recurrence, Local; Tumor Burden

\section{INTRODUCTION}

Lymph node metastasis (LNM) is a significant factor associated with disease recurrence in patients with papillary thyroid carci-

\footnotetext{
- Received March 2, 2015

Revised April 22, 2015

Accepted May 14, 2015

- Corresponding author: Young-Ik Son

Department of Otorhinolaryngology-Head and Neck Surgery, Samsung

Medical Center, Sungkyunkwan University School of Medicine,

81 Irwon-ro, Gangnam-gu, Seoul 06351, Korea

Tel: +82-2-3410-3579, Fax: +82-2-3410-3879

E-mail: yison@skku.edu

* The first two authors contributed equally to this study.
}

noma (PTC) [1-3]. The first echelon of nodal metastasis consists of paralaryngeal, paratracheal, and prelaryngeal nodes adjacent to the thyroid gland in the central compartment of the neck, which are generally described as level VI [4]. LNM in level VI is more frequently detected in the pathologic specimen [5], which is partly due to the limitations in the evaluation of the central neck with ultrasonography [6]. Even though LNM is an important risk factor of locoregional recurrence and the frequency of subclinical LNM is reported up to $65 \%$ even in subcentimeter PTC [7], the clinical significance of the central LNM has been debated because prophylactic central lymph node dissection (CLND) seems to have little prognostic benefit [5,8]. In addition, CLND can increase the frequency of postoperative transient hy-

Copyright (C) 2016 by Korean Society of Otorhinolaryngology-Head and Neck Surgery.

This is an open-access article distributed under the terms of the Creative Commons Attribution Non-Commercial License (http://creativecommons.org/licenses/by-nc/4.0)

which permits unrestricted non-commercial use, distribution, and reproduction in any medium, provided the original work is properly cited. 
pocalcemia [9]. In this context, the 2009 American Thyroid Association guidelines cautiously state that prophylactic CLND may be performed in cases of advanced primary tumors (T3 or T4), which is based on expert opinion [10].

Among the diverse staging or prognostic scoring systems which are available for differentiated thyroid carcinoma, the American Joint Committee on Cancer TNM classification is currently being used in the majority of clinical practice. Regarding the $\mathrm{N}$ staging system, the 7th edition classifies LNM into simple binary categories (presence vs. absence) based on anatomic location; patients having metastasis only to the first echelon (levelVI) are graded as $\mathrm{N} 1 \mathrm{a}$ and those having metastasis to the lateral cervical and/or mediastinal compartment are graded as N1b. Concepts on the extent of LNM including the numbers of metastatic lymph nodes, the size of metastatic foci, the presence of extranodal spread, and the metastatic lymph node ratio (MLNR, positive nodes to harvested nodes) are not involved in this classification system. Therefore, whether the current clinical and/or pathological $\mathrm{N}$ staging system can precisely reflect the probability of recurrence as well as disease specific survival remains an open question [11,12].

A recent study proposed the number of LNM as an alternative prognostic factor to supplement the limitations of the conventional $\mathrm{N}$ staging system of the thyroid gland [13]. A MLNR was proven as a significant prognostic indicator in the other organ tumors including gastric [14], pancreatic [15], and colorectal carcinomas [16]. Recently, the significance of the MLNR has also been suggested in well-differentiated thyroid carcinomas $[17,18]$. However, to the best of our knowledge, no study has investigated the clinical implications of MLNR in the central neck of the papillary thyroid microcarcinoma (PTMC) patients.

\section{MATERIALS AND METHODS}

\section{Patient selection}

From the Thyroid Cancer Registry of the Samsung Comprehensive Cancer Center, Seoul, Korea between the year 2005 and 2009,573 adult patients with the following criteria were included; (1) patients who received pre- and postoperative ultrasonography (US) imaging studies, (2) patients who underwent total thyroidectomy with CLND (lymph node dissections of prelaryngeal, paratracheal and pretracheal area), (3) patients whose surgical pathology proved to be a classical type of PTMC (less than $1 \mathrm{~cm}$ in size), (4) patients with a minimum follow-up period of 36 months. The patients who had preoperative lateral neck LNM (cN1b) on US were excluded from this study. The study protocol was approved by the Institutional Review Board of Samsung Medical Center, Seoul, Korea. Informed consent from patients was waived owing to the design of the medical record review.

Postoperative complication including hypocalcemia, vocal cord palsy, chyle leakage, and hematoma were checked. Hypocalcemia was defined as symptomatic hypocalcemia if there were perioral numbness, paresthesia of the hands and feet, Chvostek sign, or Trousseau sign; and as biochemical hypocalcemia if ionized serum calcium level is less than $1.0 \mathrm{mmol} / \mathrm{L}$. Permanent hypocalcemia was defined as persistent symptoms or persistent biochemical hypocalcemia later than the duration of 6 months. Pre- and postoperative vocal cord mobilities were routinely evaluated by fiberoptic or rigid laryngoscopy. Permanent vocal cord palsy was defined as persistent palsy later than the duration of 1 year.

\section{Statistical analyses}

MLNR was defines as the number of pathologically proven metastatic lymph nodes divided by harvested lymph nodes from the level VI. For the prediction of recurrence, multivariate analyses were performed with the following variables; the number of LNM, MLNR, and lymph node yield (LNY) of the central neck as well as previously well-known other prognostic factors including sex, size, multiplicity and the presence of extrathyroidal extension from the primary tumor. Locoregional recurrence was defined when postoperative US-guided fine needle aspiration biopsy confirmed a neck nodule as the recurrent PTC. For analyzing the characteristics of recurrence, sites of recurrence, and time to recurrence after surgery were checked.

Recurrence free interval (RFI) was analyzed with the KaplanMeier method. Univariate and multivariate analysis were performed with the Cox-regression model. The optimal cutoff value for MLNR in the central neck was determined using the minimum $P$-value approach with corrections by the Bonferroni method. A two-sided $P$-value $<0.05$ was considered significant. Statistical analysis was performed using the SAS ver. 9.1.3 (SAS Institute Inc., Cary, NC, USA) and R 2.13.2 (R Foundation for Statistical Computing, Vienna,Austria; http://www.R-project.org).

\section{RESULTS}

\section{Patient characteristics}

The clinicopathological characteristics of the study group are summarized in Table 1.There were 116 male $(20.4 \%)$ and $457 \mathrm{fe-}$ male patients $(79.6 \%)$, with a median age of 55 years. The median follow-up period was 47 months with an interquartile of 41 to 61 months. Pathological extrathyroidal extension (pETE) was detected in 252 cases (43.9\%). There were 61 clinically node-positive (cN1) (10.6\%) and $512 \mathrm{cN0}$ cases $(89.4 \%)$. The median size of the primary tumor was $6 \mathrm{~mm}$ with an interquartile of 4 to 10 $\mathrm{mm}$. The median LNY was 7 with an interquartile of 4 to 11 . The median level of stimulated serum thyroglobulin (sTg), which was measured at 3 months after surgery was $0.3 \mathrm{ng} / \mathrm{mL}$ with an interquartile of 0.1 to $1 \mathrm{ng} / \mathrm{mL}$.

Metastatic lymph node ratio

A recurrence event was detected in 15 of the 573 patients (2.6\%). 
Table 1. Summary of clinicopathological characteristics $(n=573)$

\begin{tabular}{lccc}
\hline Characteristic & $\begin{array}{c}\text { Overall } \\
(n=573)\end{array}$ & $\begin{array}{c}\text { Nonrecurrent } \\
\text { cases }(n=558)\end{array}$ & $\begin{array}{c}\text { Recurrent } \\
\text { cases }(n=15)\end{array}$ \\
\hline Age (yr) & $50(44-56)$ & $50(45-56)$ & $47(37-53)$ \\
Sex & & & \\
$\quad$ Male & $116(20.4)$ & $107(19.2)$ & $9(60.0)$ \\
Female & $457(79.6)$ & $451(20.8)$ & $6(40.0)$ \\
Tumor size (mm) & $6(4-8)$ & $6(4-8)$ & $7(5-8)$ \\
Multiplicity of primary tumor & $1(1-2)$ & $1(1-2)$ & $2(1-3)$ \\
Pathological ETS & & & \\
Yes & $252(43.9)$ & $250(44.9)$ & $2(13.4)$ \\
$\quad$ No & $321(56.1)$ & $308(55.1)$ & $13(86.6)$ \\
Clinically positive lymph node & & & \\
Yes (cN1) & $61(10.6)$ & $57(10.2)$ & $4(26.6)$ \\
No (cN0) & $512(89.4)$ & $501(89.8)$ & $11(83.4)$ \\
No. of LNM & $0(0-1)$ & $0(0-1)$ & $2(1-4)$ \\
LNY & $7(4-11)$ & $7(4-11)$ & $6(4-11)$ \\
MLNR & $0(0-0.2)$ & $0(0-0.2)$ & $0.4(0.2-0.7)$ \\
sTg (ng/mL ) & $0.3(0.1-1)$ & $0.3(0.1-0.9)$ & $7.2(3.8-23.5)$ \\
Follow-up period (mo) & $47(41-61)$ & $47(41-61)$ & $55(45-66)$ \\
\hline
\end{tabular}

Values are presented as median (interquartile range) or number (\%). ETS, extra thyroidal spread; LNM, lymph node metastasis; LNY, lymph node yield, the number of harvested lymph nodes; MLNR, metastatic lymph node ratio, the ratio between metastatic lymph node and harvested lymph nodes; sTg, level of postoperative stimulated serum thyroglobulin.

Table 2. Characteristics of recurrent cases $(n=15)$

\begin{tabular}{ll}
\hline Characteristic & Value \\
\hline Recurrence site & \\
Neck level IV & $9(60.0)$ \\
Neck level III & $3(20.0)$ \\
Thyroidectomy bed including neck level VI & $2(13.3)$ \\
Neck level VII (mediastinum) & $1(7.7)$ \\
Time to recurrence (mo) & $20(15-28)$ \\
\hline
\end{tabular}

Values are presented as number (\%) or median (interquartile range).

Among 15 cases, 12 cases $(80.0 \%)$ were recurred at the lateral neck (level III or IV), whereas operative field recurrence was observed in 2 cases $(13.3 \%)$. The median time to recurrence was 20 months, with an interquartile of 15 to 28 months (Table 2). Regarding RFI, univariate analysis using the Cox-regression model demonstrated that sex, multiplicity of the primary tumor, pETE, the number of LNM, MLNR, and sTg were significant variables (Table 3).

The MLNR was highly correlated with the number of LNM in the Spearman correlation analysis (correlation coefficient $=0.97$, $P<0.001)$. In contrast, the MLNR has no correlation with LNY in the Spearman correlation analysis (correlation coefficient= $0.06, P=0.116$ ). Therefore, LNY were included but the LNM number was excluded from the multivariate analysis of RFI. In multivariate analysis, multiplicity of the primary tumor $(P<$ $0.001), \operatorname{sTg}(P<0.001)$, and MLNR $(P=0.005)$ were proven to be associated with a higher risk of recurrence (Table 4 ).
Table 3. Univariate analysis for recurrence free interval

\begin{tabular}{lccc}
\hline Variable & Coefficient (SE) & HR (95\% Cl) & $P$-value \\
\hline Male sex & 0.53 & $6.08(2.10-17.07)$ & 0.001 \\
Age (yr) & 0.03 & $0.96(0.90-1.01)$ & 0.096 \\
$\begin{array}{l}\text { Multiplicity of primary } \\
\quad \text { tumor }\end{array}$ & 0.16 & $1.64(1.20-2.26)$ & 0.002 \\
Pathological ETS & 0.76 & $5.19(1.17-23.04)$ & 0.030 \\
$\quad$ Clinically positive lymph & 0.58 & $3.11(0.99-9.80)$ & 0.051 \\
$\quad$ node & & & \\
STg (ng/mL) & 0.01 & $1.11(1.10-1.14)$ & $<0.001$ \\
LNY & 0.05 & $0.98(0.90-1.10)$ & $>0.999$ \\
No. of LNM & 0.08 & $1.40(1.20-1.70)$ & $<0.001$ \\
MLNR & 0.66 & $27.98(5.80-134.36)$ & $<0.001$ \\
\hline
\end{tabular}

$\mathrm{SE}$, standard error; HR, hazard ratio; $\mathrm{Cl}$, confidence interval; ETS, extra thyroidal spread; sTg, level of postoperative stimulated serum thyroglobulin; LNY, Iymph node yield, the number of harvested lymph nodes; LNM, lymph node metastasis; MLNR, metastatic lymph node ratio, the ratio between metastatic lymph node and harvested lymph nodes.

Table 4. Multivariate analysis for recurrence free interval

\begin{tabular}{lrc}
\hline Variable & $P$-value & $\mathrm{HR}(95 \% \mathrm{Cl})$ \\
\hline Male sex & 0.013 & $4.67(1.38-15.79)$ \\
Age (yr) & 0.706 & $0.99(0.93-1.05)$ \\
Multiplicity of primary tumor & $<0.001$ & $2.02(1.42-2.88)$ \\
Pathological ETS & 0.365 & $2.11(0.42-10.62)$ \\
Clinically positive lymph node & 0.147 & $0.39(0.11-1.40)$ \\
sTg (ng/mL) & $<0.001$ & $1.10(1.07-1.14)$ \\
LNY & 0.827 & $1.01(0.89-1.15)$ \\
MLNR & 0.005 & $11.21(2.12-59.40)$ \\
\hline
\end{tabular}

$\mathrm{HR}$, hazard ratio; $\mathrm{Cl}$, confidence interval; ETS, extra thyroidal spread; sTg, level of postoperative stimulated serum thyroglobulin; LNY, lymph node yield, the number of harvested lymph nodes; MLNR, metastatic lymph node ratio, the ratio between metastatic lymph node and harvested lymph nodes.

\section{Cutoff value for predicting recurrence}

Cutoff values of the MLNR showed significant predictive power between 0.44 and 0.50 . The lowest cutoff value was 0.44 (adjusted hazard ratio [HR], 8.86; 95\% confidence interval [CI], 1.4952.58; $P<0.001)$ and the cutoff value which had the highest HR was 0.45 (adjusted HR, 9.78; 95\% CI, 1.68-57.09; $P=0.002$ ).

\section{Postoperative complications}

Of the 573 in the study, temporary hypocalcemia developed in 167 patients (29.1\%). Permanent hypocalcemia developed in 6 patients $(1.0 \%)$. Transient vocal cord paralysis or paresis developed in 30 patients $(5.2 \%)$ and permanent palsy in 7 patients $(1.2 \%)$. Minor chyle leakage occurred in 5 patients $(0.8 \%)$, which were easily controlled with conservative management including a week of low-fat diet or a fat-free diet. Immediate postoperative hematoma was observed in 4 patients $(0.6 \%) ; 3$ from the anterior jugular vein of the subplatysmal flap and 1 form the superior thyroid artery. 


\section{DISCUSSION}

Despite the excellent overall survival of patients with PTMC regardless of the extent of initial treatment, LNM is an important prognostic factor which frequently results in increased diseaserecurrence [19]. Therapeutic CLND for patient with clinically involved central or lateral neck lymph nodes should accompany total thyroidectomy to provide clearance of disease from the central neck [10]. However, there is no current consensus regarding the optimal subsequent treatment when the LNM in the central neck is firstly confirmed after the surgery. Therefore, there were continuing debates on the needs for the completion thyroidectomy, further complete CLND, or postoperative radioactive iodine therapy.

Lee et al. [13] recently addressed the prognostic implications of the number of LNM of the central neck; the number of central LNM correlates with the well-known adverse prognostic indicators including tumor size, extrathyroidal extension, and lateral neck LNM. However, they analyzed the number of LNM in relation to indirect prognostic variables including sTg, age, sex, or tumor size rather than direct therapeutic outcomes including recurrence or survival. Another recent study revealed that both the number of central LNM and $\mathrm{N}$ stage are significant prognostic indicators to predict recurrences in PTMC patients. In a subgroup analysis of PTMC patients having no lateral neck LNM, number of central LNM $(\geq 4)$ remained as a meaningful predictor for recurrence [20]. Besides the number LNM $(\geq 3)$ in the central neck, the size of metastatic foci $(\geq 0.2 \mathrm{~cm})$ and the presence of desmoplasia in the metastatic lymph nodes were highly associated with recurrence-free survival [21].

To minimize the confounding effects of the primary tumor and to exclusively focus on the prognostic significance of the LNM, our study included only the patient populations with PTMC, who received total thyroidectomy with CLND. Both the absolute number and the relative ratio of LNM showed a statistical significance in the prediction of disease-recurrence from the univariate analysis $(P<0.001)$. To verify this association, multivariate analysis was performed by adjusting the effects of the following variables; sex, age, multiplicity of the primary tumor, pETE, clinical N staging, LNY, and postsurgical sTg level. The number of LNM exhibited its prognostic value in predicting disease-recurrence with an adjusted HR of 1.28 (95\% CI, 1.01 to $1.62 ; P=0.033)$. However, we failed to retrieve a meaningful cutoff value regarding the number of central LNM, which indirectly indicates a weak correlation between the number of LNM and disease-recurrence (data not shown).

Besides the well-established current concepts of size, number, and extracapsular spread in the nodal staging system, MLNR was proposed as an alternative prognostic marker in gastric, colorectal and pancreatic cancers [14-16]. Similarly, Lang et al. [18] reported that a higher MLNR is associated with a higher level of sTg, which might imply persistent or recurrent disease; if the MLNR in central neck was $>0.67$, the chance of having detectable sTg was 36.64 times higher than if the MLNR was $<0.3$. However, this seems to be an over-simplified risk-stratification rather than a validated cutoff value to predict locoregional recurrence. Regarding the MLNR for predicting recurrence, both total MLNR $(\geq 0.7)$ and central neck MLNR $(\geq 0.86)$ were strongly associated with recurrence of PTC after initial operation even though the study was retrospectively performed in a small group of patients $(n=69)$ analysis [22]. By analyzing the Surveillance Epidemiology and End Results (SEER) Database, Beal et al. [17] showed that increased MLNR was associated with decreased survival in patients with well-differentiated thyroid carcinoma. Another recent SEER database analysis of 10,995 cases of PTC having LNM indicated that MLNR $\geq 0.42$ is a strong determinant of disease-specific mortality [23].

In multivariable analysis adjusting for other variables, the MLNR was proven as a significant prognostic indicator for the prediction of disease-recurrence with an adjusted hazard ratio of 11.21 (95\% CI, 2.12 to 59.40; $P=0.005)$. However, the recurrence events were small (15 cases, $2.6 \%$ ) and therefore, the confidence interval widely ranged (2.12 to 59.40$)$, which is one of the limitations of this study.

For the prediction of locoregional recurrence, the lowest cutoff value of the MLNR was $0.44(P<0.001 ; \mathrm{HR}, 8.86)$. The cutoff value which has the highest HR was $0.45(P=0.002$; HR, 9.78). However, careful interpretation of these cutoff values of MLNR should be conducted when a small number of lymph nodes is harvested. Regarding the pattern of recurrence, the majority of recurrence was found at the lateral neck; 9 patients $(60.0 \%)$ in level IV and 3 patients $(20.0 \%)$ in level III. Recurrence at the operative bed was found only in 2 cases $(13.3 \%)$. The median period to the recurrence after thyroidectomy was 20 months with an interquartile of 15 to 28 months. A skip metastasis, defined as lateral LNM without central LNM, occurs in only a minority of patients with PTMC [24]. The concept of the step-wise progression of LNM originating from the central to the lateral neck has largely been accepted [25]. Therefore, a higher MLNR in the central neck may imply the possibility of subsequent or subclinical LNM in the lateral neck.

There is a philosophical limitation of this study. Before the year 2009, the authors' institution preferred total thyroidectomy with CLND as the treatment choice for the patients with PTMC, especially when the patient is male, older than 45 years, and has a familial history of PTC. As a result, we could enroll a unique patient cohort in this study, who received unusually aggressive treatments for their low-risk PTMC; total thyroidectomy with CLND. However, relatively uniform and consistent treatments could paradoxically provide important and valuable information about the significance of MLNR. Other limitations include the retrospective study design, a relatively short-term follow-up period, and a limited number of recurrent cases. In addition, the data analyzed in this study was retrieved only from a single in- 
stitution. All of these shortcomings might result in selection bias, thereby weakening the statistical power.

In this study, we proved that MLNR in the central neck has a predictive value for locoregional recurrence. When the MLNR is higher than 0.44 , there is an increased risk of locoregional recurrence with an HR of $8.86(P<0.005)$. Because most of the recurrence was observed in the lateral neck $(80 \%)$, a careful follow-up evaluation of the lateral neck is warranted in these patients. Even though prophylactic CLND is not recommended as a treatment guideline for cNO PTMC, ipsilateral CLND combined with hemithyroidectomy is not infrequently performed as an initial surgical management of PTMC in many countries including Korea. In this context, the concept of MLNR with a cutoff value of 0.44 may supplement the decision making for the further treatment. For example, confirming the MLNR of the central neck in a permanent or frozen biopsy may be helpful in decision makings for the further surgical resection including total thyroidectomy, completion thyroidectomy, or contralateral CLND.

\section{CONFLICT OF INTEREST}

No potential conflict of interest relevant to this article was reported.

\section{REFERENCES}

1. Ito Y, Jikuzono T, Higashiyama T, Asahi S, Tomoda C, Takamura Y, et al. Clinical significance of lymph node metastasis of thyroid papillary carcinoma located in one lobe. World J Surg. 2006 Oct;30(10): 1821-8.

2. Lundgren CI, Hall P, Dickman PW, Zedenius J. Clinically significant prognostic factors for differentiated thyroid carcinoma: a populationbased, nested case-control study. Cancer. 2006 Feb;106(3):524-31.

3. Podnos YD, Smith D, Wagman LD, Ellenhorn JD. The implication of lymph node metastasis on survival in patients with well-differentiated thyroid cancer.Am Surg. 2005 Sep;71(9):731-4.

4. Edge SB, Compton CC. The American Joint Committee on Cancer: the 7th edition of the AJCC cancer staging manual and the future of TNM.Ann Surg Oncol. 2010 Jun;17(6):1471-4.

5. Wada N, Duh QY, Sugino K, Iwasaki H, Kameyama K, Mimura T, et al. Lymph node metastasis from 259 papillary thyroid microcarcinomas: frequency, pattern of occurrence and recurrence, and optimal strategy for neck dissection. Ann Surg. 2003 Mar;237(3):399-407.

6. Stulak JM, Grant CS, Farley DR, Thompson GB, van Heerden JA, Hay ID, et al. Value of preoperative ultrasonography in the surgical management of initial and reoperative papillary thyroid cancer. Arch Surg. 2006 May;141(5):489-94.

7. Kouvaraki MA, Shapiro SE, Fornage BD, Edeiken-Monro BS, Sherman SI, Vassilopoulou-Sellin R, et al. Role of preoperative ultrasonography in the surgical management of patients with thyroid cancer. Surgery. 2003 Dec;134(6):946-54.

8. Noguchi S, Yamashita H, Uchino S, Watanabe S. Papillary microcarcinoma. World J Surg. 2008 May;32(5):747-53.

9. Palestini N, Borasi A, Cestino L, Freddi M, Odasso C, Robecchi A. Is central neck dissection a safe procedure in the treatment of papillary thyroid cancer? Our experience. Langenbecks Arch Surg. 2008 Sep;393(5):693-8.

10. American Thyroid Association (ATA) Guidelines Taskforce on Thyroid Nodules and Differentiated Thyroid Cancer, Cooper DS, Doherty GM, Haugen BR, Kloos RT, Lee SL, et al. Revised American ThyroidAssociation management guidelines for patients with thyroid nodules and differentiated thyroid cancer.Thyroid. 2009 Nov;19(11): 1167-214.

11. Wada N, Nakayama H, Suganuma N, Masudo Y, Rino Y, Masuda M, et al. Prognostic value of the sixth edition AJCC/UICCTNM classification for differentiated thyroid carcinoma with extrathyroid extension. J Clin Endocrinol Metab. 2007 Jan;92(1):215-8.

12. Ito Y, Miyauchi A, JikuzonoT, HigashiyamaT,Takamura Y, Miya A, et al. Risk factors contributing to a poor prognosis of papillary thyroid carcinoma: validity of UICC/AJCC TNM classification and stage grouping. World J Surg. 2007 Apr;31(4):838-48.

13. Lee YS, Lim YS, Lee JC,Wang SG, Kim IJ, Lee BJ. Clinical implication of the number of central lymph node metastasis in papillary thyroid carcinoma: preliminary report. World J Surg. 2010 Nov;34(11): 2558-63.

14. Celen O, Yildirim E, Berberoglu U. Prognostic impact of positive lymph node ratio in gastric carcinoma. J Surg Oncol. 2007 Aug; 96(2): 95-101.

15. Slidell MB, Chang DC, Cameron JL, Wolfgang C, Herman JM, Schulick RD, et al. Impact of total lymph node count and lymph node ratio on staging and survival after pancreatectomy for pancreatic adenocarcinoma: a large, population-based analysis. Ann Surg Oncol. 2008 Jan;15(1):165-74.

16. Ceelen W, Van Nieuwenhove Y, Pattyn P. Prognostic value of the lymph node ratio in stage III colorectal cancer: a systematic review. Ann Surg Oncol. 2010 Nov;17(11):2847-55.

17. Beal SH, Chen SL, Schneider PD, Martinez SR. An evaluation of lymph node yield and lymph node ratio in well-differentiated thyroid carcinoma.Am Surg. 2010 Jan;76(1):28-32.

18. Lang BH, Wong KP, Wan KY, Lo CY. Significance of metastatic lymph node ratio on stimulated thyroglobulin levels in papillary thyroid carcinoma after prophylactic unilateral central neck dissection. Ann Surg Oncol. 2012 Apr;19(4):1257-63.

19. Hay ID, Hutchinson ME, Gonzalez-LosadaT, McIver B, Reinalda ME, Grant CS, et al. Papillary thyroid microcarcinoma: a study of 900 cases observed in a 60-year period. Surgery. 2008 Dec;144(6):980-7.

20. Lee J, Song Y, Soh EY. Central lymph node metastasis is an important prognostic factor in patients with papillary thyroid microcarcinoma. J Korean Med Sci. 2014 Jan;29(1):48-52.

21. Cho SY, Lee TH, Ku YH, Kim HI, Lee GH, Kim MJ. Central lymph node metastasis in papillary thyroid microcarcinoma can be stratified according to the number, the size of metastatic foci, and the presence of desmoplasia. Surgery. 2015 Jan;157(1):111-8.

22. Schneider DF, Mazeh H, Chen H, Sippel RS. Lymph node ratio predicts recurrence in papillary thyroid cancer. Oncologist. 2013;18(2): 157-62.

23. Schneider DF, Chen H, Sippel RS. Impact of lymph node ratio on survival in papillary thyroid cancer. Ann Surg Oncol. 2013 Jun;20(6): 1906-11.

24. Chung YS, Kim JY, Bae JS, Song BJ, Kim JS, Jeon HM, et al. Lateral lymph node metastasis in papillary thyroid carcinoma: results of therapeutic lymph node dissection. Thyroid. 2009 Mar;19(3):241-6.

25. Machens A, Hauptmann S, Dralle H. Lymph node dissection in the lateral neck for completion in central node-positive papillary thyroid cancer. Surgery. 2009 Feb;145(2):176-81. 